\title{
Inelastic Constitutive Properties and Shear Localization in Tennessee Marble
}

\author{
D.J. Holcomb, Sandia National Laboratories* \\ and \\ J.W. Rudnicki, Northwestern University
}

May, 1999

\section{Introduction}

Shear bands and faults are ubiquitous features of brittle rock deformation at a variety of length scales. Despite the prevalence of these features, understanding of their inception remains rudimentary. Laboratory experiments suggest a casual association of localization of deformation (faulting) with peak stress, but more detailed examination reveals that localization can precede or follow the peak.

Rudnicki and Rice (1975, hereafter abbreviated as RR) have suggested a theory of the inception of localization as a bifurcation or nonuniqueness of the solution for homogeneous deformation. They predict a strong dependence of localization on deformation state. In particular, they predict that localization can occur prepeak for deformation states near deviatoric pure shear and does not occur until well after peak for axisymmetric compression. This prediction is roughly in accord with the true triaxial experiments of Mogi $(1967,1971)$. More recently, Ord et al. (1991) and Wawersik et al. (1991) have reported observations of localization prior to peak stress in plane strain experiments.

The predictions of $R R$ depend strongly on the constitutive properties of the rock and detailed comparison has been impeded by inadequate knowledge of those properties. Even the idealized constitutive model used by RR requires knowledge

- Sandia is a multiprogram laboratory operated by Sandia Corporation, a Lockheed Martin Company, for the United States Department of Energy under Contract DE-AC04-94Al8500. 


\section{DISCLAIMER}

Portions of this document may be illegible in electronic image products. Images are produced from the best available original document. 
of the evolution of the constitutive properties with inelastic deformation that is not readily obtainable from the typical axisymmetric compression test. Although it is conceptually advantageous to consider inelastic deformation at fixed mean stress, the mean stress changes throughout the axisymmetric compression test.

In this paper, we present a synthesis of a number of axisymmetric compression tests to extract a detailed implementation of the constitutive framework used by $R R$. The resulting constitutive relation is then used to predict the response for plane strain. Conditions for localization of deformation derived by RR are evaluated for both plane strain and axisymmetric compression.

\section{Theoretical Background}

\subsection{Constitutive Framework}

The constitutive framework used here is that of RR who generalized the type of relation used for metal plasticity. In particular, they included pressure dependence of the yield condition and inelastic volume change.

The yield condition is the surface in the space of stress components $\sigma_{i j}$ that is the boundary of those stress states for which the response is elastic; for those stress states on the yield surface, the response is inelastic. In general, the yield surface is not fixed but evolves with one or more parameters that characterize accumulated inelastic (or plastic) deformation. It will be convenient to decompose the stress into a deviatoric part $s_{i j}$ and a mean normal contribution $\sigma$

$$
\sigma_{i j}=s_{i j}+\sigma \delta_{i j}
$$

where $\delta_{i j}(=1$, if $i=j,=0$, if $i \neq j)$ is the Kronecker delta, $\sigma=(1 / 3) \sigma_{k k}$, and the repeated subscript implies summation. Plastic strain increments are similarly decomposed

$$
d \epsilon_{i j}^{p}=d \epsilon_{i j}^{p}+\frac{1}{3} \delta_{i j} d \epsilon^{p}
$$

The yield condition is assumed to be of the following form

$$
\bar{\tau}-f\left(\sigma, \bar{\gamma}^{p}\right)=0
$$

where $\bar{\tau}=\sqrt{\frac{1}{2} s_{i j}, x_{i j}}$, and the accumulated plastic shear strain

$$
\bar{\gamma}^{p}=\int_{0}^{t} \sqrt{2 \dot{\epsilon}_{i j}^{p} \dot{\epsilon}_{i j}^{p}} d t
$$


is the sole parameter used to keep track of the history of inelastic deformation. (The superposed dot indicates the derivative with respect to time or any monotonically increasing parameter). The requirement that the stress state remain on the yield surface for continuing inelastic deformation is the consistency condition:

$$
d \bar{\tau}-\mu d \sigma-h d \bar{\gamma}^{p}=0
$$

where $\mu\left(\sigma, \bar{\gamma}^{p}\right)=\partial f / \partial \sigma$ is a friction coefficient, and $h\left(\sigma, \bar{\gamma}^{p}\right)=\partial f / \partial \bar{\gamma}^{p}$ is a plastic hardening modulus.

Expressions for the plastic portion of the strain increments are specified by the flow rule

$$
d \epsilon_{i j}^{p}=\frac{\partial \Gamma}{\partial \sigma_{i j}} d \lambda
$$

where $\Gamma=\bar{\tau}-g\left(\sigma . \bar{\gamma}^{p}\right)$ is the plastic potential function and $d \lambda \geq 0$. Taking the deviatoric part of (6) and substituting for the plastic potential yields

$$
d \epsilon_{i j}^{p}=d \lambda \frac{s_{i j}}{2 \bar{\tau}}
$$

Using (7) in (4) reveals that $d \lambda=d \bar{\gamma}^{p}$ and the inelastic volume strain can be written as

$$
d \epsilon^{p}=-\beta d \bar{\gamma}^{p}
$$

where $\beta\left(\sigma, \bar{\gamma}^{p}\right)=\partial g / \partial \sigma$ (and the minus sign appears because stresses and strains are taken to be positive in compression). From the consistency condition, the increment in accumulated plastic shear strain is given by

$$
d \bar{\gamma}^{p}=\frac{d \bar{\tau}-\mu d \sigma}{h\left(\sigma, \bar{\gamma}^{p}\right)}
$$

Since $d \bar{\gamma}^{p} \geq 0$, (9) applies for increments tending to make $d \bar{\tau}>\mu d \sigma(h>0)$; for increments tending to make $d \bar{\tau} \leq \mu d \sigma(h>0), d \bar{\gamma}^{p}=0$, and the material unloads elastically. Substituting the definitions for $\bar{\tau}$ and $\sigma$, and recombining the deviatoric and volumetric plastic strain increments yields

$$
d \epsilon_{i j}^{p}=\frac{1}{h}\left(\frac{s_{i j}}{2 \bar{\tau}}-\frac{1}{3} \beta \delta_{i j}\right)\left(\frac{s_{k l}}{2 \bar{\tau}}-\frac{1}{3} \mu \delta_{k l}\right) d \sigma_{k l}
$$




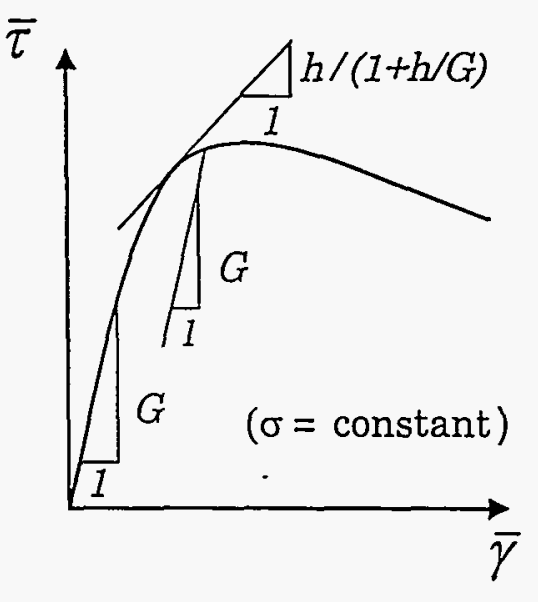

a.

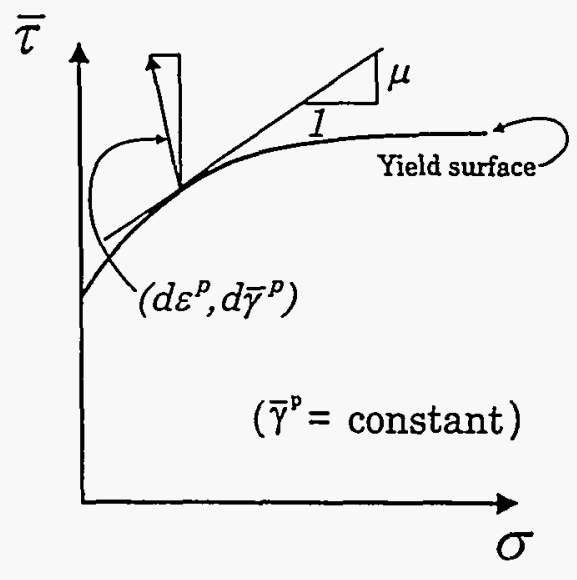

b.

Figure 1: Geometric interpretation of the parameters $h$ (a) and $\beta$ and $\mu$ (b). The slope of the vector $\left(d \epsilon^{p}, d \bar{\gamma}^{p}\right)$ is $-\beta$.

Minus signs in (10 are the result of choosing compressive stress to be positive, opposite to the choice made in RR. The total strain increment is the sum of (10) and an elastic increment. For isotropic elasticity the latter is:

$$
d \epsilon_{i j}^{e}=\frac{1}{2 G}\left(d \sigma_{i j}-\frac{\nu}{1+\nu} d \sigma_{k k} \delta_{i j}\right)
$$

where $G$ is the shear modulus and $\nu$ is Poisson's ratio. Figure 1a shows the geometric interpretation of $h$ in a sketch of the shear stress versus shear strain at constant mean stress. Figure lb shows that $\mu$ is the local slope of the yield surface in the space $\bar{\tau}$ versus $\sigma$ and that the plastic strain increment vector $\left(d \epsilon^{p}, d \bar{\gamma}^{p}\right)$ would be perpendicular to the yield surface if $\beta$, the negative of the ratio of the volume and shear plastic strain increments, were equal to $\mu$.

\subsection{Shear Localization}

$R R$ proposed that faulting could be described as bifurcation from homogeneous deformation. That is, they examined the conditions for which non-uniform deformation in a planar band was an alternative to homogeneous deformation. Formation of the band was required to be consistent with a continuous velocity field and equations expressing continuing equilibrium. These requirements result in a condition depending on the parameters of the constitutive relation and the orientation of the band. Then RR determined the orientation of the band for which this 
condition is first met. For the constitutive framework introduced in the preceding section, this condition can be expressed as a critical value of the hardening modulus at which localization is first possible. The result is

$$
\frac{h_{\text {cr }}}{G}=\frac{(1+\nu)}{9(1-\nu)}(\beta-\mu)^{2}-\frac{1}{2}(1+\nu)\left[N+\frac{1}{3}(\beta+\mu)\right]^{2}
$$

where $\mu, \beta, G$ and $\nu$ are as defined earlier and $N$ is the negative of the intermediate principal deviatoric stress divided by $\bar{\tau}$. (As discussed in more detail by $R R$, the expression (12) neglects terms of order $\bar{\tau} / G$; typically, these are small.)

The parameter $N$ specifies the deviatoric stress state and ranges from $-1 / \sqrt{3}$ for axisymmetric extension, through 0 for deviatoric pure shear to $1 / \sqrt{3}$ for axisymmetric compression, using the convention that compressive stress is positive. The maximum value of $h_{c r}$ occurs for $N=-(\beta+\mu) / 3$ and is positive if $\mu \neq \beta$. $\mathrm{RR}$ evaluate the condition (12) for various constant values of $N, \mu$, and $\beta$, as have a number of others, but, in general, these parameters evolve during a program of loading. In general, the value of $N$ evolves during plane strain, but for an incompressible material, $N=0$ during plane strain. For $N=-\beta / 3$, the intermediate principal value of the inelastic strain rate is zero and, to the neglect of elastic strains, this corresponds to plane strain.

\section{Implementation for Tennessee Marble}

To implement the constitutive framework, it is necessary to choose a specific yield function $f\left(\bar{\gamma}^{p}, \sigma\right)$ and plastic potential $g\left(\sigma, \bar{\gamma}^{p}\right)$ (or, equivalently, the dependence of the dilatancy factor on $\sigma$ and $\bar{\gamma}^{p}$ ). A form that is tractable, yet suffices to describe the Tennessee marble data adequately, is the following:

$$
\bar{\tau}=\tau_{0}+f_{1}\left(\bar{\gamma}^{p}, \sigma\right)+f_{2}(\sigma)
$$

where

$$
f_{1}\left(\bar{\prime}^{\prime} \cdot \sigma\right)=\left(h_{0}+h_{\infty}\right) \gamma_{0}^{p}(\sigma) \arctan \left(\frac{\bar{\gamma}^{p}}{\gamma_{0}^{p}(\sigma)}\right)-h_{\infty} \bar{\gamma}^{p}
$$

and

$$
f_{2}(\sigma)=\mu_{0} \min \left(\sigma, \sigma_{0}\right)
$$


represents the initial yield surface and its mean stress dependence. In (13-15), $\tau_{0}, h_{0}, h_{\infty}, \mu_{0}$, and $\sigma_{0}$ are positive constants. The normalizing strain $\gamma_{0}^{p}(\sigma)$ is assumed to depend linearly on the mean stress

$$
\gamma_{0}^{p}(\sigma)=\gamma_{00}+\gamma_{01}\left(\sigma / \sigma_{0}\right)
$$

In the fitting procedure we actually used the slightly different form $\bar{\gamma}_{01}=\gamma_{01} / \sigma_{0}$. Because $\sigma_{0}$ is a fitted parameter, this form must be used in reproducing the data fit to get the same functional relationship.

The hardening modulus and friction coefficient can be derived using (13) and the expressions following (5). The results are

$$
\begin{aligned}
h\left(\bar{\gamma}^{p}, \sigma\right) & =\frac{h_{0}+h_{\infty}}{1+\left[\bar{\gamma}^{p} / \gamma_{0}^{p}(\sigma)\right]^{2}}-h_{\infty} \\
\mu\left(\bar{\gamma}^{p}, \sigma\right) & =\mu_{0} \mathrm{H}\left(\sigma_{0}-\sigma\right) \\
& +{ }^{\circ} \frac{\left(h_{0}+h_{\infty}\right)}{\sigma_{0}}\left\{\arctan \left(\frac{\bar{\gamma}^{p}}{\gamma_{0}^{p}(\sigma)}\right)-\frac{\bar{\gamma}^{p} / \gamma_{0}^{p}(\sigma)}{1+\left[\bar{\gamma}^{p} / \gamma_{0}^{p}(\sigma)\right]^{2}}\right\}
\end{aligned}
$$

where $\mathrm{H}(s)$ is the Heaviside step function. Because $h(0, \sigma)=h_{0}$, rather than becoming unbounded, the slope of the shear stress versus shear strain curve (at constant $\sigma$ ) is discontinuous at $\bar{\gamma}^{p}=0$. In reality, the transition from purely elastic behavior is smooth but this feature of the model is inconsequential.

At peak shear stress, $h=0$ and from (17) the equivalent plastic strain at peak shear stress $\left(\bar{\gamma}^{\text {peut }}\right)$, is found to be

$$
\bar{\gamma}^{\text {peak }}(\sigma)=\sqrt{h_{0} / h_{\infty}} \gamma_{0}^{p}(\sigma) .
$$

Note that because the equivalent plastic shear strain at peak shear stress depends on the mean stress. the locus of the peak shear stress in the $\bar{\tau}$ vs. $\sigma$ plane is not a yield surface as is commonly assumed. The peak shear stress, for constant mean stress, is given by

$$
\bar{\tau}_{p}=\bar{\tau}_{p}^{0}+T \sigma
$$

where

$$
\bar{\tau}_{p}^{0}=\tau_{0}+\gamma_{0},\left\{\left(h_{0}+h_{\infty}\right) \arctan \sqrt{\left(h_{0} / h_{\infty}\right)}-h_{\infty} \sqrt{\left(h_{0} / h_{\infty}\right)}\right\}
$$

and

$$
T=\mu_{0} \mathrm{H}\left(\sigma_{0}-\sigma\right)+\left[\frac{\gamma_{01}}{\sigma_{0}}\right]\left\{\left(h_{0}+h_{\infty}\right) \arctan \sqrt{\left(h_{0} / h_{\infty}\right)}-h_{\infty} \sqrt{\left(h_{0} / h_{\infty}\right)}\right\} .
$$


Figure 2 illustrates the graphical interpretation of the parameters. Figure 2a sketches the shear stress $\bar{\tau}$ as a function of the equivalent plastic shear strain $\bar{\gamma}^{p}$ for constant values of the mean stress $\sigma$. Figure $2 \mathrm{~b}$ sketches yield surfaces (surfaces of constant $\bar{\gamma}^{p}$ ) in the $\bar{\tau}$ vs. $\sigma$ plane.

An expression for the dilatancy factor was developed by writing the accumulated plastic volume strain as $\epsilon^{p}=E\left(\bar{\gamma}^{p}, \sigma\right)$. Because $\epsilon^{p}$ depends on stress path, it cannot, in general. be represented in a functional form such as this. The introduction of $E$ is, however, simply a device for representing the data for the particular stress path of axisymmetric compression and only the increments of plastic strain enter the constitutive formulation. An increment of inelastic volume strain is given by

$$
d \epsilon^{p}=\frac{\partial E}{\partial \bar{\gamma}^{p}} d \bar{\gamma}^{p}+\frac{\partial E}{\partial \sigma} d \sigma
$$

The negative of the coefficient in the first term is the dilatancy factor. (Note that $E$ is related to the flow potential by $\partial g / \partial \sigma=-\partial E / \partial \bar{\gamma}^{p}$.) The second term represents inelastic compaction, that is, inelastic volume strain due to mean stress. Here this term is deleted since the $R R$ formulation assumes that all inelastic volume change is related to inelastic shearing. A more general representation would include a term representing inelastic compaction, although there is no reason to expect that the coefficients of the two terms can necessarily be expressed as partial derivatives of a single function. A form of the function $E($ gamma $\left.a^{p}, \sigma\right)$ suggested by the data is

$$
E\left(\tau \gamma^{p}, \sigma\right)=\left[\beta_{\infty}-B\left(\sigma / \sigma_{0}\right)\right] \bar{\gamma}^{p}+\left(\beta_{\infty}-\beta_{0}\right) c(\sigma) \arctan \left\{\frac{\bar{\gamma}^{p}}{c(\sigma)}\right\}
$$

where

$$
c(\sigma)=c_{0}-c_{1}\left(\sigma / \sigma_{0}\right)
$$

and $\beta_{0} . \beta_{\infty}, c_{0}, c_{1}$. and $B$ are constants. The dilatancy factor is given by

$$
\beta(\sigma . \therefore)=-B\left(\sigma / \sigma_{0}\right)+\beta_{\infty}-\frac{\beta_{\infty}-\beta_{0}}{1+\left(\bar{\gamma}^{p} /\left[c_{0}-c_{1}\left(\sigma / \sigma_{0}\right)\right]\right)^{2}}
$$

Thus, the dilatancy factor at $\bar{\gamma}^{p}=0$ is $\beta_{0}-B\left(\sigma / \sigma_{0}\right.$ and $\beta$ approaches $\beta_{\infty}-$ $B\left(\sigma / \sigma_{0}\right)$ as $\bar{\gamma}^{p}-\infty$. Figures $3 \mathrm{a}$ and $3 \mathrm{~b}$ sketch $\beta$ and $\epsilon^{p}=E$ as a function of $\bar{\gamma}^{p}$ for two values of mean stress. Numerical values for these coefficients are in Table 1. 


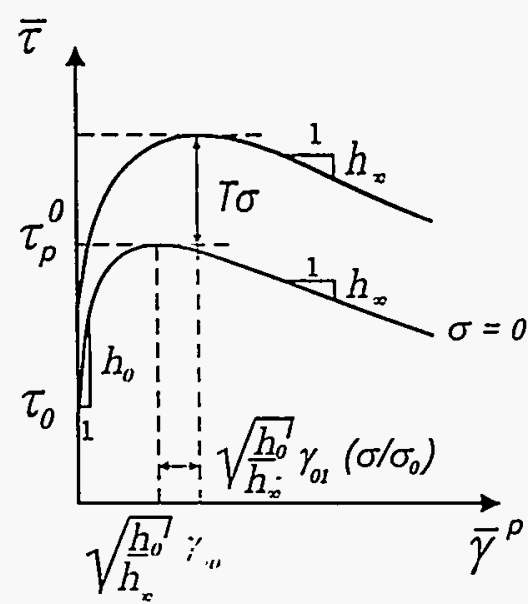

a.

Figure 2: Key parameters used to model the dependence of $\mu$ and $h$ on $\bar{\gamma}_{p}$ (a) and $\sigma(\mathrm{b})$.

\section{Determination of Model Parameters}

Determining the model parameters is a several step process, beginning with acquisition of a datal set, determining $\tau, \sigma, \bar{\gamma}^{p}$ and $\epsilon^{p}$ and finally fitting the model parameters to the processed data set. Experimental results from conventional triaxial tests on $5 \mathrm{~cm}$ diameter cylinders of Tennessee marble were analyzed to obtain $\bar{\gamma}^{p}$ and $\epsilon^{p}$. The elastic strains were removed by using a least squares fitting process to simultaneously determine $\mathrm{G}$ and $\nu$ from the initial portions of the loading curves. Although $G$ and $\nu$ change with inelastic deformation, the change is small for Tennessee marble and was neglected here (see Table 1 for values). Twelve experiments, conducted at confining pressures from 0 to $100 \mathrm{MPa}$, were analyzed to produce a data set consisting of values for $\bar{\tau}, \sigma, \bar{\gamma}^{p}$ and $e^{p}$. Our goal was to find values for the model parameters listed in Table 1 which would minimize a suitable measure of the difference between the experimental and modeled values for $\bar{\tau}$ and $\epsilon^{p}$. A simplex algorithm was used to solve the minimization problem.

Although the downhill simplex method is not a particularly efficient algorithm, it is appealing for solving minimization problems because it is easy to implement, requires no differentiation and readily allows inclusion of constraints. Nelder and Mead (1965) first described the downhill simplex method, but a more accessible reference may be Numerical Recipes (Press et al., 1986, pp. 289). A simplex in two dimensions is a triangle (three vertices), in three dimensions a tetrahe- 

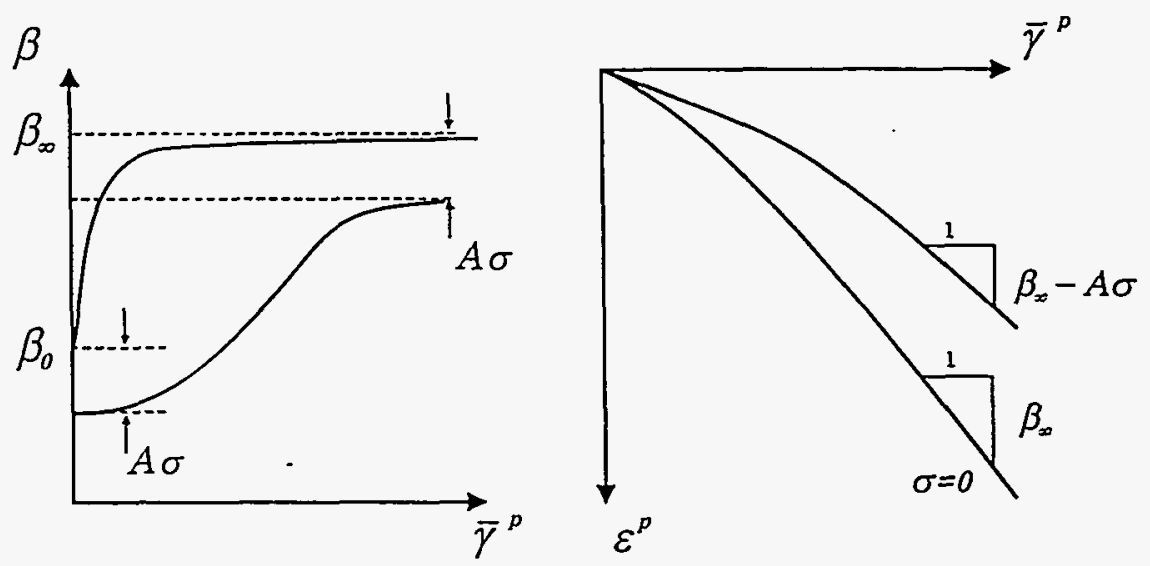

a.

b.

Figure $3: \beta$ as a function of $\bar{\gamma}_{p}(\mathrm{a})$ and parameters used in the model $(\mathrm{b})$

dron (four vertices) and so on to an object with $\mathrm{N}+1$ vertices in $\mathrm{N}$ dimensions corresponding to the $\mathrm{N}$ unknowns of the minimization problem. Each vertex corresponds to an $\mathrm{N}$-tuple of possible values for the sought-after set of parameters that minimize the appropriate measure of error. In our problem, the unknowns are the 12 parameters listed in Table 1 . The error function to be minimized was the L2 norm of the difference between the experimental values of $\bar{\tau}$ or $\epsilon^{p}$ and the corresponding values calculated from Equations 13 and 22. The procedure was implemented with the constraint that $\mu<\sqrt{3}$, the slope of the $\bar{\tau}$ versus $\sigma$ trajectory in a conventional triaxial test.

To begin the process, $\mathrm{N}+1$ starting vectors are chosen that define the vertices of the initial simplex in the $\mathrm{N}$-dimensional space of unknowns. At each vertex, the error function is evaluated, by calculating the value of $\bar{\tau}$ for the several hundred experimental data points, using the experimental values of $\bar{\gamma}^{p}$ and $\sigma$ and the $\mathrm{N}$ tuple of parameter values that are the vertex coordinates. Then the simplex shape is modified to move it towards regions of the parameter space that give lower values for the error functions. The simplest movement is away from the vertex with the highest value of the error function, accomplished by reflecting that vertex across to the other side of the simplex. In two dimensions the process is easily visualized as an amoeba-like series of stretchings, contractions and crawling that moves the triangular simplex as a whole towards smaller and smaller error values. When a minimum lies within the simplex, further movement ceases to lower the error and a series of contractions are instituted that shrink the simplex until some specified convergence criterion is met. There is no guarantee that the minimum 


\begin{tabular}{|l|l|}
\hline Parameter & Value \\
\hline$G_{r}$ & $30 \mathrm{GPa}$ \\
\hline$\nu$ & 0.30 \\
\hline$h_{0}$ & $68.27 \mathrm{GPa}$ \\
\hline$h_{\infty}$ & $0.62 \mathrm{GPa}$ \\
\hline$\mu_{0}$ & 0.39 \\
\hline$\gamma_{00}$ & $3.84 \times 10^{-5}$ \\
\hline$\gamma_{01}$ & $3.61 \times 10^{-4}$ \\
\hline$\sigma_{0}$ & $68.57 \mathrm{MPa}$ \\
\hline$\tau_{0}$ & $34.72 \mathrm{MPa}$ \\
\hline$\beta_{0}$ & 0.43 \\
\hline$\beta_{\infty}$ & 1.49 \\
\hline$C_{0}$ & $2.37 \times 10^{-4}$ \\
\hline$c_{1}$ & $3.71 \times 10^{-3}$ \\
\hline$B$ & $3.32 \times 10^{-2}$ \\
\hline
\end{tabular}

Table 1: Numerical Values of Coefficients

is not just a local minimum, so it is standard practice to restart the simplex at different regions of the parameter space to determine whether the same minimum is found.

Two separate minimizations were carried out: one to obtain the parameters necessary to describe the hardening modulus and the friction coefficient and the other for the parameters in the model of the dilatancy parameter. As Figures 2 and 3 show, the parameters could be found by choosing values from individual tests at special points. However, fitting all of the triaxial test data avoided overemphasizing any one test or portion of a test. Another approach would have been to model the hardening modulus, friction factor and dilatancy parameter directly. This would have required differentiating the experimental stress-strain curves, which would inevitably produce a noisy data set to be fit. By modeling $\bar{\tau}$ and $\epsilon^{p}$ as functions of $\bar{i}$ and $\sigma$, we were able to carry out the differentiations required to obtain $h, \mu$, and 3 on smooth analytic functions.

Once determined, the parameters in Table 1 can be used to calculate the response for different stress paths by substituting the expressions for $\mu, \beta$ and $h$ into (10) and numericilly integrating. A comparison of experiment and calculations for all of the fitted triaxial tests is shown in Figure 4. This comparison confirms the suitability of the lorms adopted for $\Gamma$ (6) and $E$ (22). Calculations were checked 
by comparing the results from a direct integration of Equation 10 with the output from a radial return algorithm. Care was required in the direct integration to keep the stress on the yield surface. Frequent correction steps had to be included to eliminate a tendency to wander away from the yield surface. Once properly corrected, the agreement between the two solution algorithms was excellent.

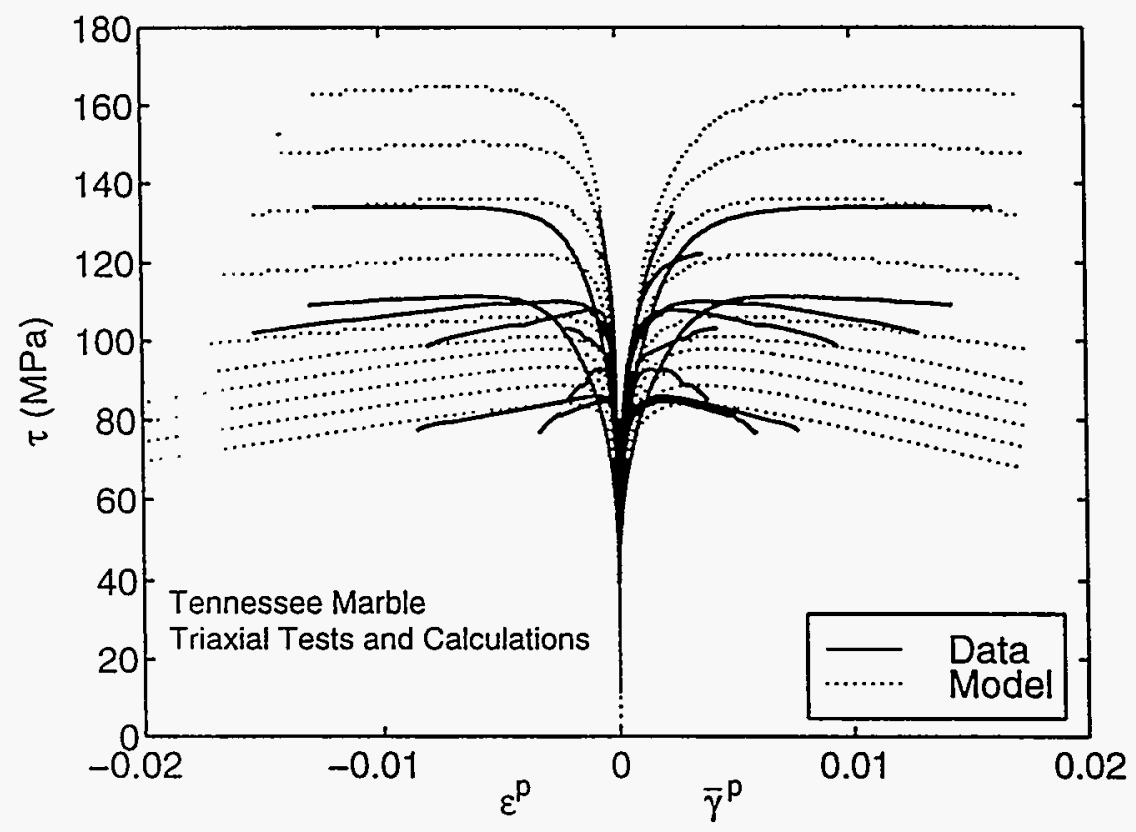

Figure 4: Comparison of experimental results used to derive the model parameters with the stresses and strains calculated from using the fitted parameters.

Calculated yield surfaces for several values of $\bar{\gamma}^{p}$ are shown in Figure 5a and shear stress versus shear strain at several values of mean stress are shown in Figure $5 b$. Also shown in Figure 5a is the peak stress as a function of mean stress. As discussed earlier, the curve of peak stress cuts across the yield surfaces (not shown) and, hence, is not itself a yield surface. This result is not model-dependent, as any model that predicts the observed plastic shear strains would give essentially the same result. Since the peak stress is, in general, neither a yield surface nor the stress at which localization occurs, the prominence given this parameter may be more due to its e:lse of observation than any fundamental significance. 


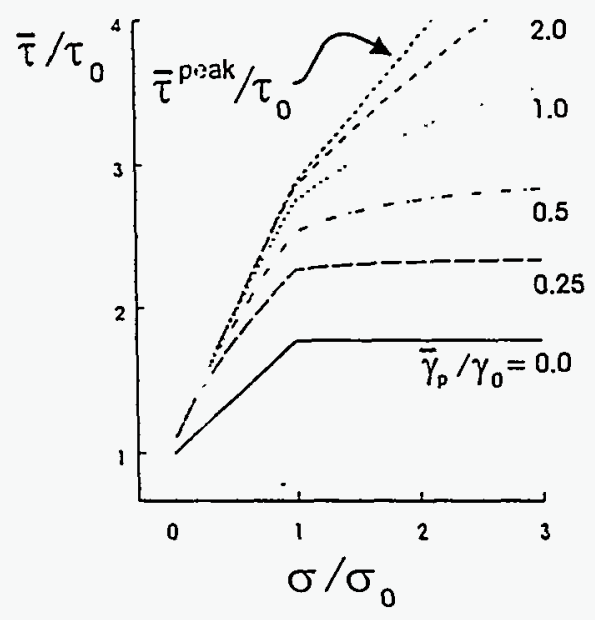

a.

Figure 5: Results of modeling yield surfaces (a) and shear stress as a function of plastic shear strain $\bar{\gamma}^{p}$ at constant mean stress (b). The curve marked $\bar{\tau}^{\text {peak }} / \tau_{0}$ in (a) shows peak stress as a function of $\sigma$. From numerical results the locus of peak stresses crosses the yield surfaces, indicating that peak stresses do not constitute a yield surface.

\section{Localization Under Axisymmetric Compression}

Using the constitutive relations described earlier, we have examined the localization criterion (Equation 12) for axisymmetric compression tests. Figure 6 shows the evolution of the hardening modulus (17) and of the critical hardening modulus needed for localization (12) for the cases $\sigma_{22}=\sigma_{33}=5$ and $20 \mathrm{MPa}$. Results for other confining stresses are similar: the hardening modulus decreases but never becomes sufficiently negative to equal the critical value. The minimum attainable value of $h$ in the constitutive model is negative $\left(-h_{\infty}\right.$, see Equation (17)), as is the critical value predicted for localization. However, $h_{c r}$ is predicted to be a substantial fraction of the elastic shear modulus $G$, exceeding the possible values for $h$.

As discussed by RR (and by Rice (1976)), the strongly negative values of $h_{\text {crit }}$ predicted for localization in axisymmetric compression are related to the overly stiff response of al smooth yield surface model to the abrupt change in the pattern of deformation required for localization. Essentially, localization into a planar band is similar to a plane-strain mode of deformation; when the pre-localization field is axisymmetric, the formation of a band requires an abrupt change in the 


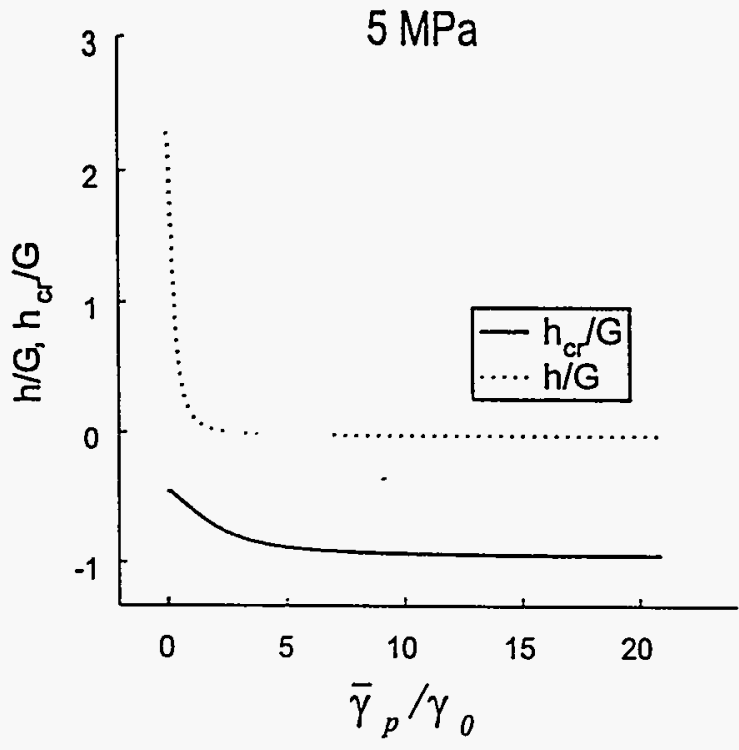

a.

Figure 6: Calculation of the critical hardening modulus and the material hardening modulus, plotted as a function of normalized plastic shear strain for triaxial load paths at confining pressures $\sigma_{22}=\sigma_{33}=5$ and $20 \mathrm{MPa}$, showing that the localization criterion is never met. The calculation was done using $G=30 \mathrm{GPa}$ and $\nu=0.3$

ratio of components of inelastic strain increments. RR note that the overly stiff response to this abrupt change predicted by smooth yield surface models is alleviated by models that have a vertex at the current stress point. This class is predicted for a wide range of microstructural models (Hill, 1967) and evidence for the formation of a yield surface vertex has been observed in compression-torsion tests on Tennessee marble (Olsson, 1992). Because post-test examination of the samples revealed localization, in the form of through-going fractures, it appears that a more elaborate constitutive formulation, possibly including a yield surface with a corner. is needed to accurately predict localization in axisymmetric compression.

\section{Simulations of Plane Strain}

We have also used the constitutive relation to simulate the results of a plane strain test (zero strain in the $x_{2}$ direction) with constant in-plane compressive stress 
$\left(\sigma_{33}=20 \mathrm{MPa}\right)$. Results for the stresses, $\sigma_{11}$ and $\sigma_{22}$ versus $e_{11}$ are shown in Figure 7. Dashed lines are the results obtained from the model and solid lines are experimental data. Note that the hardening in plane strain is augmented, by comparison with that in axisymmetric compression, because of the more rapid increase of mean stress in plane strain.

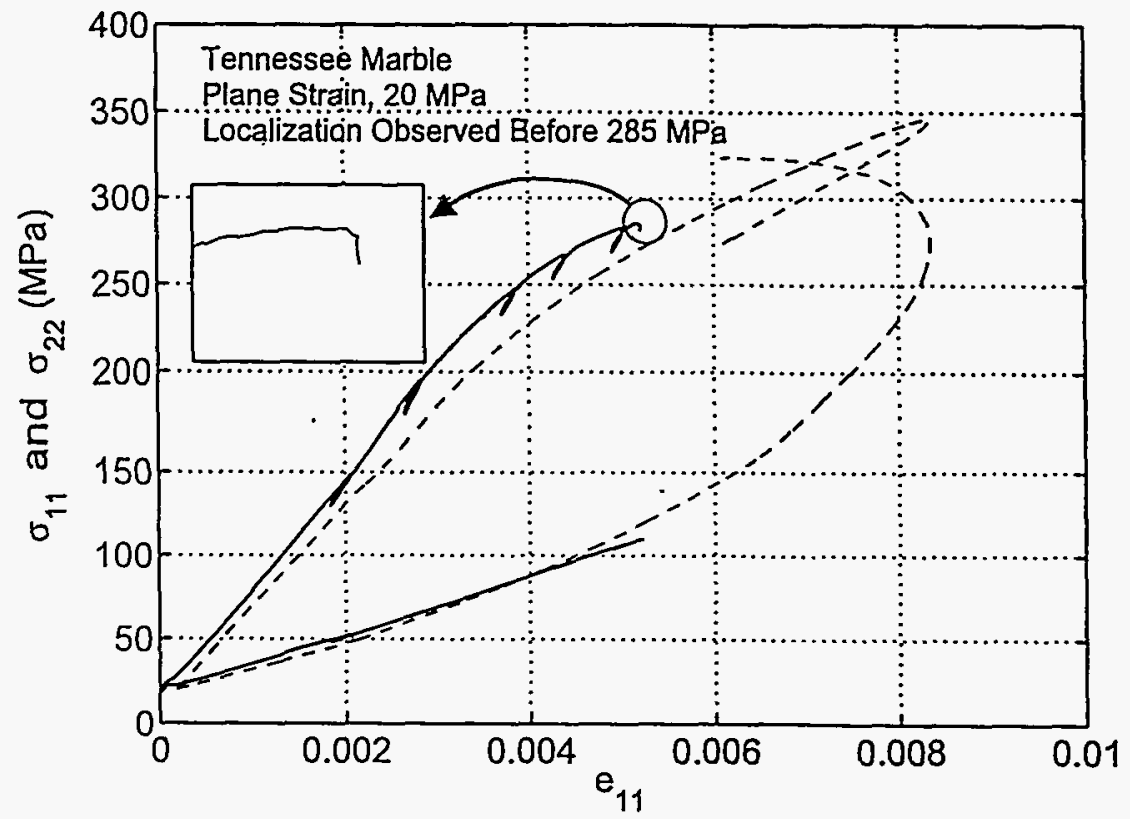

Figure 7: Comparison of the calculated (dashed lines) and experimental (solid lines) stresses as a function of total strain $e_{11}$ for a plane strain test on Tennessee marble. Using the convention that compressive stress is positive, $\sigma_{11}$ and $\sigma_{22}$ are the maximum and intermediate compressive stresses, respectively.

Modeling reproduced the stresses quite well. Note that the test included unloading loops (the small ticks off the main load line) that were not included in the modeling. Model calculations were extended to much higher values than were observed, in order to reach predicted localizaton. Experimental data indicate a nearly linear response of $\sigma_{22}$ as would be expected if the material remained elastic. Figure 8 confirms that, experimentally, elastic strain along the out-of-plane axis was small. Because total strain $e_{22}=\epsilon_{22}^{e}+\epsilon_{22}^{p}=0$ was constrained to be exactly zero (plane strain), $\epsilon_{22}^{e}=-\epsilon_{22}^{p}$. Thus a small elastic strain component implies an equally small plastic strain component of opposite sign. In contrast 
to the experimental results, the modeled results for $e_{22}$ in Figure 8 show a large compressive, elastic strain, and thus a large, extensive, plastic strain.

The discrepancy appears to be caused by the way in which dilatancy is modeled (Equation (8)): An increment of inelastic deviatoric strain, in any direction, contributes to $d \bar{\gamma}^{p}$ and causes equal increments of inelastic normal strain in all directions. In actuality, dilatancy in the $x_{2}$-direction is likely to be suppressed because shearing occurs principally in the $x_{1}-x_{3}$ plane and because the opening of microcracks in the $x_{2}$ - direction is opposed by the increasing normal stress in this direction. Inclusion of this effect would require an anisotropic model and indicates the difficulties of constructing a constitutive model from (even many) results for a single deformation state.

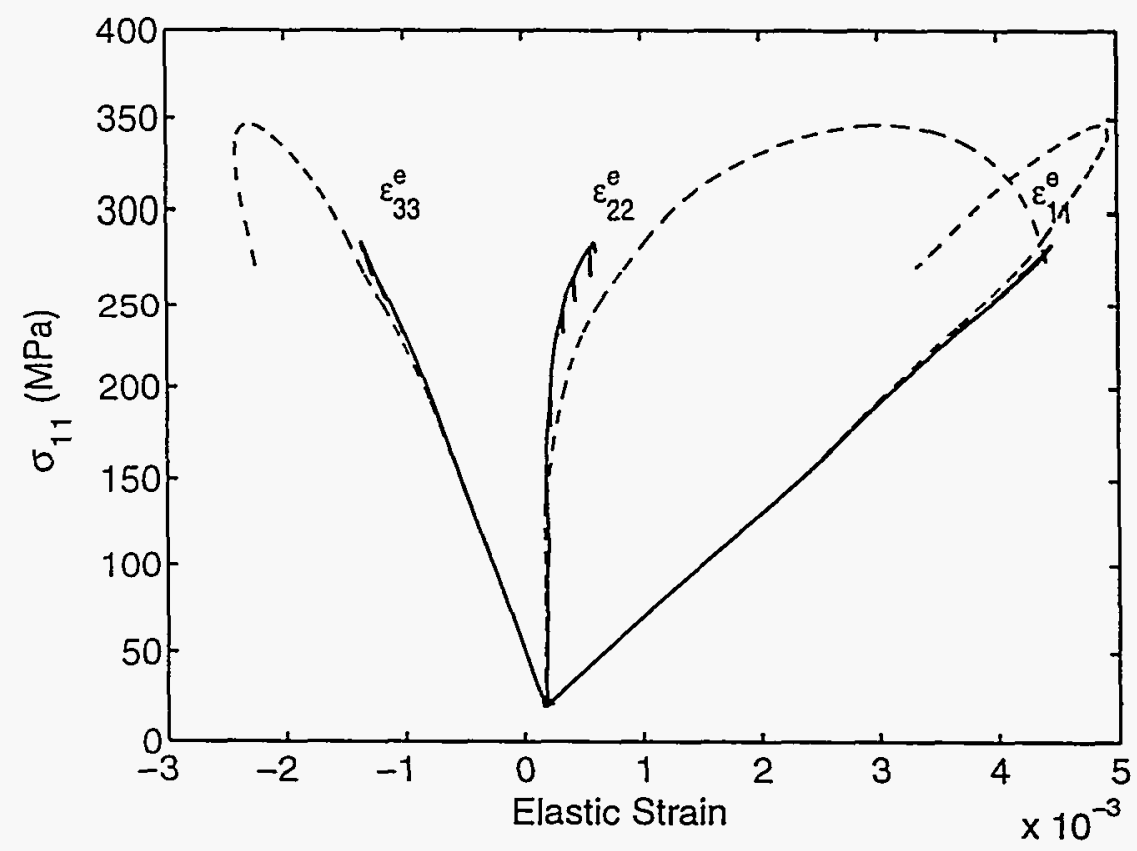

Figure 8: Comparison of calculated (dashed lines) and experimental (solid lines) elastic strains vs $\sigma_{11}$ for a plane strain test on Tennessee marble. The in-plane strain components $\left(\epsilon_{11}^{e}\right.$ and $\left.\epsilon_{33}^{e}\right)$ were well described by the model, but the out-ofplane component $\left(\epsilon_{22}^{e}\right)$ was not.

Evolution of the constitutive parameters $h, h_{c r}, \beta$ and $\mu$ as a function of $\bar{\gamma}^{p}$ during the course of the plane strain test is shown in Figure 9. Values resulting from the integration of the model (10) are shown as dashed lines. An alternative approach is to calculate $\bar{\gamma}^{p}, \epsilon^{p}$, and $\sigma$ from the test data and then, using Equations 


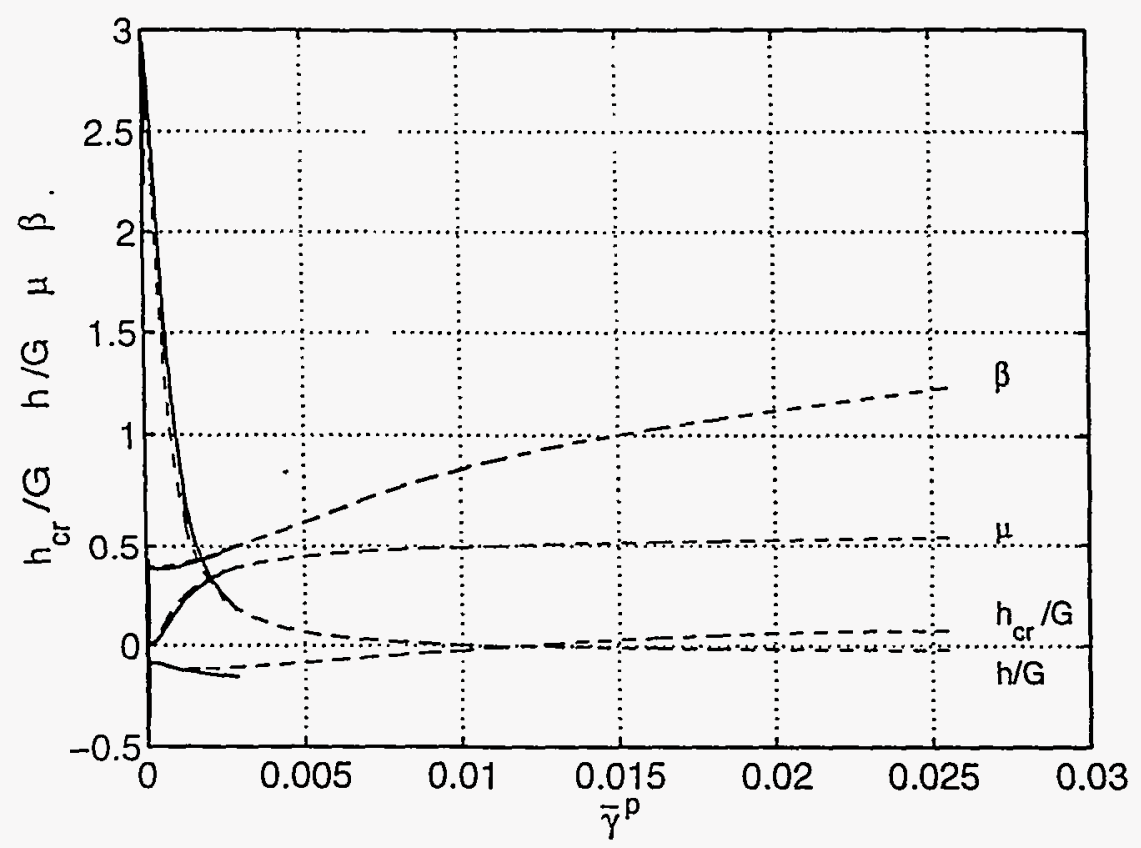

Figure 9: Comparison of calculated (dashed lines) and experimental (solid lines) values of $h, h_{c r}, \beta$ and $\mu$ as a function of $\bar{\gamma}^{p}$ for a plane strain test on Tennessee marble.

12,17 and 24 , calculate $h, h_{c r}, \beta$ and $\mu$ directly (solid lines). Values obtained this way are, in a sense, the experimental values, to be compared with the model values obtained by integration. Agreement was quite good over the range of plastic shear strain reached in the experiment. At the end of the experimental loading, discussed in detail below, $h / G$ was still positive at about 0.2 , while $h_{c r}$ was negative and decreasing. The dilatancy parameter $\beta$ increased by about a factor of 3 from 0.4 to about 1.2, while the friction parameter, after an initial sharp increase from near zero, remained relatively constant at 0.5 .

The test was conducted using servo-hydraulic control of the load frame to maintain the strain rate constant for $e_{33}$, the strain in the minimum compresive stress direction, ensuring that stability was maintained as localization was occurring. As a result, it was possible to observe what we interpreted to be the occurrence of localization; $\sigma_{11}$, the maximum compressive stress began to decrease spontaneously (unload) as $e_{33}$ continued to increase (see enlarged portion of Figure 7.) This implies the onset of a process that caused the strain rate in the minimum compressive stress (3) direction to increase rapidly. Only by reducing $\sigma_{11}$ 
and thus its contribution to $\dot{e}_{33}$, under the automatic control of the servo-hydraulic system, was it possible to maintain the specified strain rate. Localization is the likely cause of the change in behavior that occurred at $\sigma_{11}=285 \mathrm{MPa}$.

This observation is compared with the prediction of localization made by plotting the evolution of the hardening modulus (divided by $G$ ) and the evolution of the critical hardening modulus (given by (12)) against $\bar{\gamma}^{p}$ (Figures 9 and enlarged in 10). Model results indicate that the critical hardening modulus was initially negative but increased during the test, resulting in the satisfaction of the localization criterion $h=h_{c r}$ when the decreasing $h=h_{c r} \approx 0$. This is in contrast to the case for axisymmetric compression where it was found that the predicted localization criterion required a large negative $h$.

The evolution of $h_{c r}$ is due to changes of $\mu, \beta$ and the deviatoric stress state parameter, $\lambda$. which is plotted against the total axial strain in Figure 11. At the onset of loading, $N \approx 0.15$ as required for the elastic solution to plane strain loading. During the test, $N$ evolves toward axisymmetric compression and then back towards pure shear $(N=0)$.

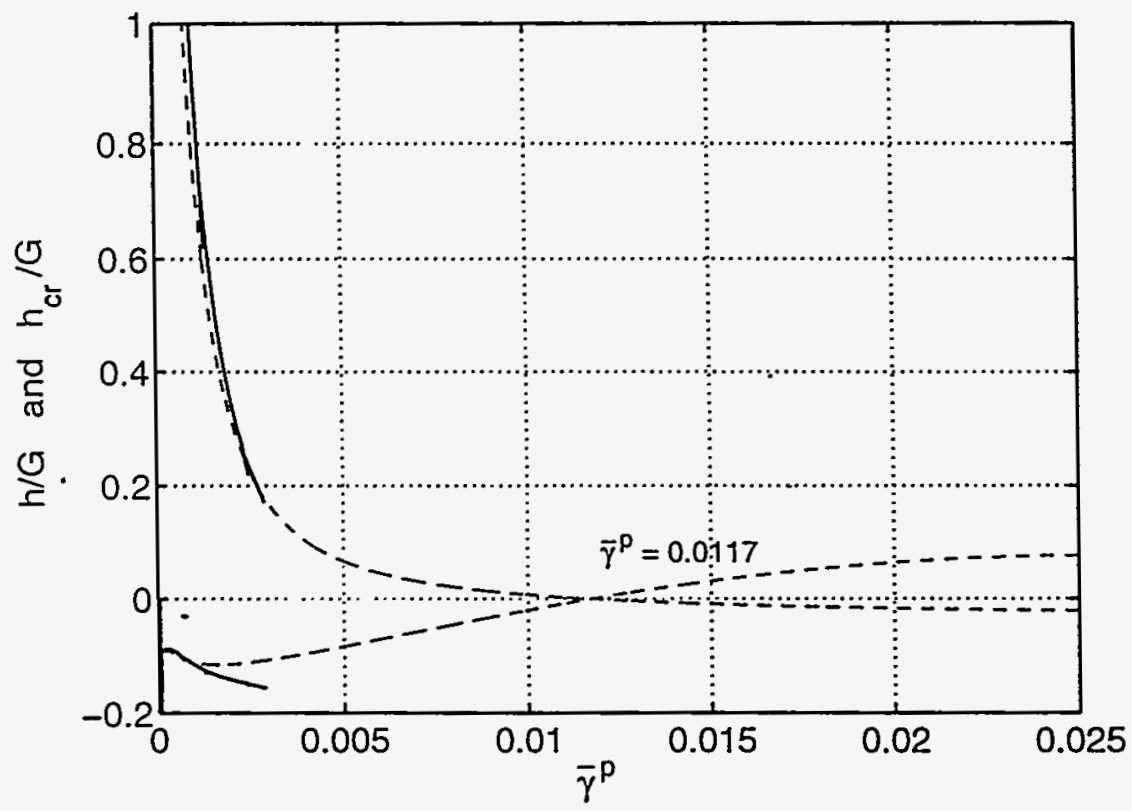

Figure 10: From modeling, localization is predicted to occur for plane strain conditions when $h=h_{c r}=-2.5 \times 10^{-4}$ (dashed lines) at $\bar{\gamma}^{p}=0.0117$. This is significantly different from the experimental results (solid lines) which were interpreted to show localization at approximately $h=0.2$. 


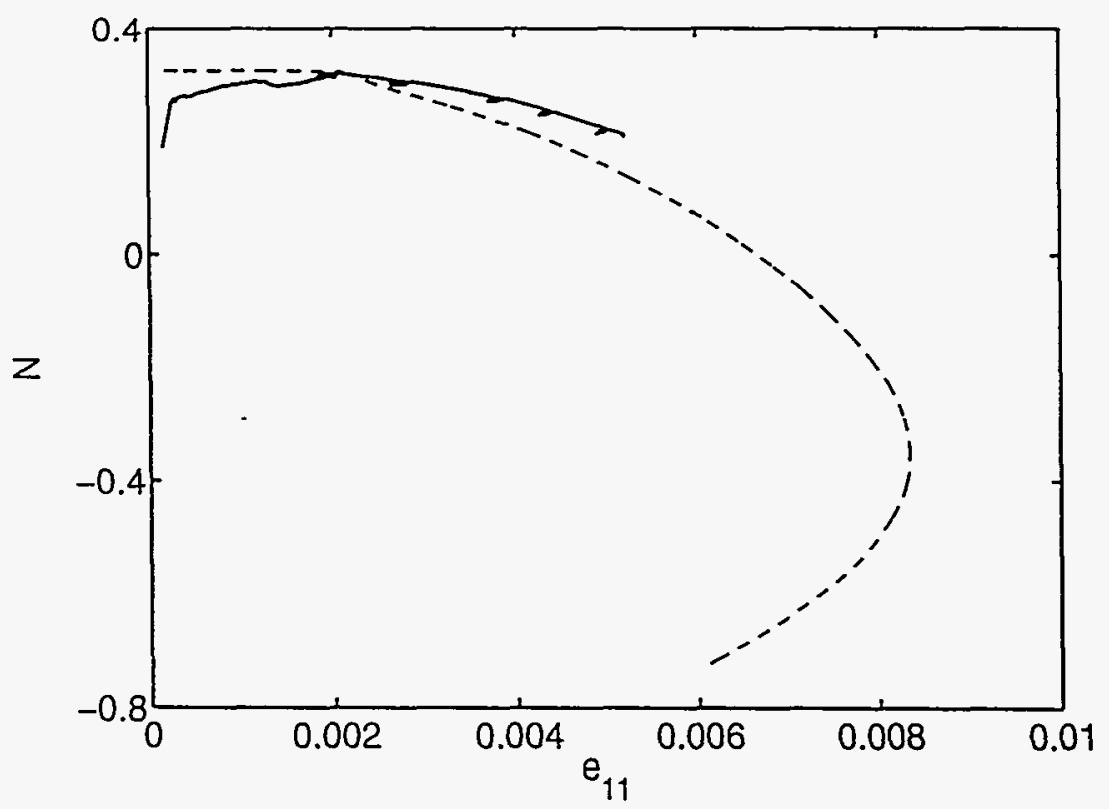

Figure 11: Evolution of $N$ during the course of a plane strain test as a function of $\epsilon_{11}$, the strain along the maximum compressive stress axis, showing the evolution towards an axisymmetric stress state and back towards pure shear.

It is misleading to draw conclusions about the sign of $h$ from the local shape of stress-strain curves at localization as was done by Ord et. al, 1991. They argued that the observation of localization during increasing stress implied that $h$ was positive, i.e. that localization had occurred "pre-peak". This argument is only valid for constant mean stress tests. For a constant mean stress test $(\dot{\sigma}=0) h=0$ implies that the peak shear stress and the peak of the stress-strain curves has been reached. In the case where $\sigma$ is not constant, which includes most commonly performed tests, it is not possible to determine the sign of $h$ at any given point by examining stress-strain curves. For example, as Figure 12 shows, at the predicted localization point $\left(\bar{\gamma}^{p}=0.0117\right)$, the shear stress is still increasing, even though $h=-2.5 \times 10^{-4}$ is already slightly negative (see Figure 10). Only by a detailed calculation, similar to the one carried out here, can the value of $h$ be determined, allowing an assessment of whether localization occurred for positive or negative $h$, that is, in the hardening or softening regime.

A simpler, constructed example, devoid of the computational complexity, serves to illuminate this important point; localization can be predicted to occur under rising load, i.e.. the slope of $\sigma_{11} v s . \epsilon_{11}$ is positive, even when $h<0$. In particular, 


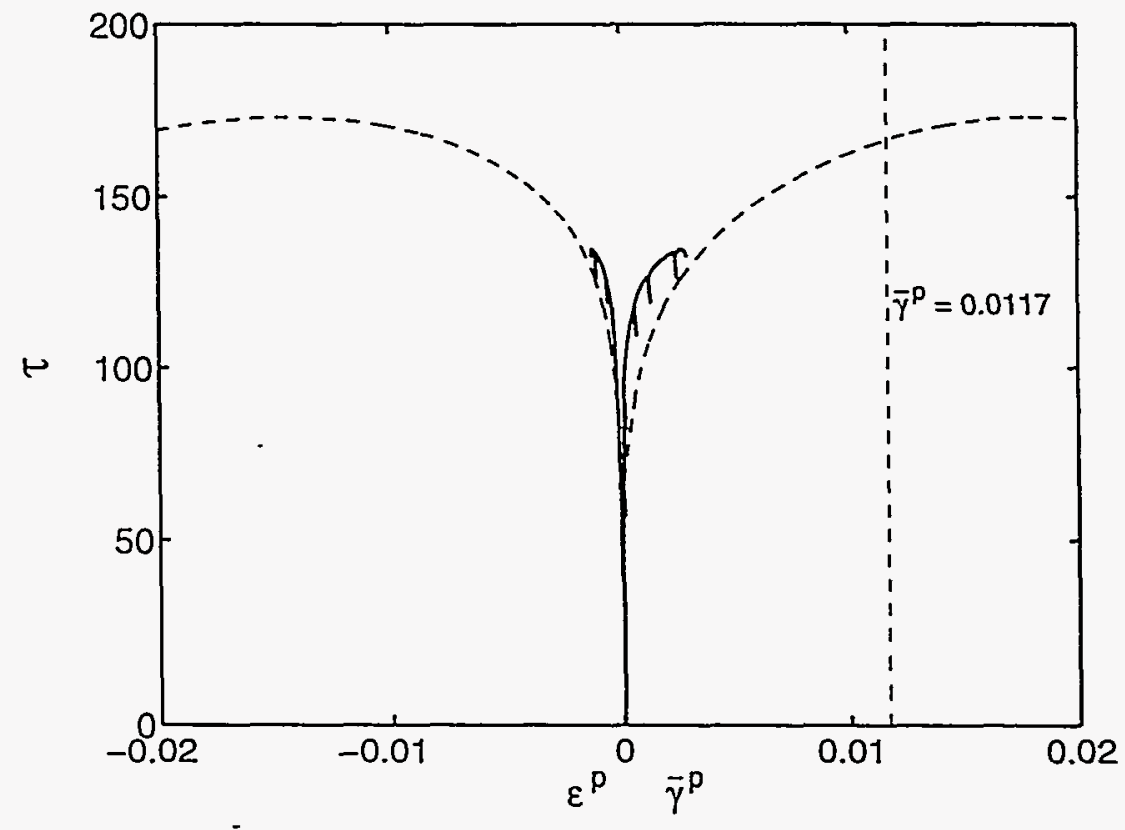

Figure 12: Comparison of calculated (dashed lines) and experimental (solid lines) plastic strains vs $\bar{\gamma}^{p}$ for a plane strain test on Tennessee marble. As a result of changing mean stress, the stress-strain curve appears to show hardening, even though $h$ was already slightly negative, in the softening regime.

because of the rapid increase of mean stress in the plane strain test, it is possible for $h$ to be negative while the slope of $\sigma_{11}$ vs $\epsilon_{11}$ is positive. Such an example is shown in Figure 13.

Here, the yield condition is given by

$$
\bar{\tau}=\tau_{0}-0.02 G \bar{\gamma}^{p}-0.7 \sigma
$$

In addition, it is assumed that $\beta=0$ and the lateral confining stress is zero. Thus, $h$ has the constant value $-G / 50$, and $\mu$ has the constant value 0.7 . The resulting pure shear response (constant $\sigma$ ) is bilinear: an elastic portion with slope $G$ until the shear stress reaches $\tau_{0}$ followed by a descending portion with slope $-G / 49$. Similarly, the modeled axial stress versus axial strain response in axisymmetric compression is also bilinear. For Poisson's ratio $\nu=0.2$, the slope is $E=2.4 G$, until the axial stress reaches $5 \tau_{0} / \sqrt{3}$, and then the slope is $-12 G / 115$.

For plane strain, however, the response is nonlinear. As shown in Figure 13a, even though $h=-G / 50<0$ as soon as inelastic deformation occurs, the curve 
of $\sigma_{11} v s . \epsilon_{11}$ continues to rise until peak load (indicated by the triangle). In this example, $h_{c r} / G=0.0653$ for deviatoric pure shear $(N=0)$ and -0.3127 for axisymmetric compression. Thus, localization is predicted to occur for deviatoric pure shear but not for axisymmetric compression. For plane strain, $h_{c r} / G$ evolves with deformation (Figure 13b) because of the changing value of $N$ (Figure 13c). Localization is predicted to occur at the point indicated by the box, when $h_{c r}$ has risen to equal the constant value of $h=-G / 50$ (Figure 13b). The increase of the critical value of $h_{c r}$ in this case is due entirely to the evolution of the deviatoric stress state from near axisymmetric compression $(N=1 / \sqrt{3})$, for which $h_{c r}$ is very negative, towards deviatoric pure shear $(N=0)$, for which $h_{c r}$ is less negative. Thus, observation of localization under rising load in plane strain does not necessarily mean that $h$ is positive.

\section{Conclusion}

Beginning from experimental data for axisymmetric compressive testing on Tennessee marble, we determined all the parameters required for the RR theory of localization as a function of plastic shear strain and mean stress. Numerical techniques were used to integrate the incremental plastic strain expressions for comparison with original axisymmetric test data and for prediction of the results of plane strain tests. A feature that emerges from the data analysis is that the peak stress is not a yield surface as is conventionally assumed. This result is modelindependent; it will be the case whenever the data indicate that the inelastic strain at peak stress depends on the mean stress.

In addition to the basic calculations of stress and strain, predictions of the localization criterion were made and compared with experimental results. Under axisymmetric loading, localization is observed to occur post-peak in experiments as is predicted by the bifurcation theory. The predicted values of $h$ required for localization are, however, so negative that the modeled axisymmetric tests never reached the localization criterion. A more sophisticated treatment, including the effects of vertices on the yield surface, appears to be necessary to predict accurately the strain (or hardening modulus) at localization in the axisymmetric case (Rudnicki and Rice, 1975).

Localization was predicted to occur when $(h \approx 0)$ for the plane strain test $\left(\dot{e}_{22}=0\right)$ that was modeled. The predicted localization stress was significantly higher than the experimental observation of spontaneous unloading (decreasing $\sigma_{11}$ ) that we interpreted to coincide with the formation of a shear zone. Results of 
modeling the test and from a simpler, constructed example, show that the sign of $h$, the hardening modulus cannot be determined from examination of the stressstrain curves. An important prediction of RR is the possibility of pre-peak $(h>0)$ localization. Testing this prediction in plane strain requires the use of a model to calculate $h$.

Modeling captured the behavior of $\sigma_{11}, \sigma_{22}, e_{11}$ and $e_{33}$ well, but did not do as well with $e_{22}$. The poor prediction of the out-of-plane strain behavior is attributed to an over prediction of $\epsilon_{22}^{p}$ due to absence of anisotropy in the model.

\section{Acknowledgements}

Financial support for the work of DJH was provided by the U. S. Dept. of Energy, Office of Basic Energy Sciences, Geosciences Research Program through a grant to Sandia National Laboratories.

Financial support for the work of JWR was provided by the U. S. Dept. of Energy, Office of Basic Energy Sciences, Geosciences Research Program through Grant No. DE-FG02-93ER14344/09 to Northwestern University.

\section{References}

[1] Hill, R. The essential structure of constitutive laws for metal composites and polycrystals, J. Mech. Phys. Solids, Vol. 15, 79-95, 1967.

[2] Ord, A., I.Vardoulakis, and R. Kajewski, Shear band formation in Gosford Sandstone, Int. J. Rock Mech. \& Mining Science, Vol. 28, 397-409, 1991.

[3] Rice, J. R., The localization of plastic deformation, in Proceedings of the 14th International Congress of Theoretical and Applied Mechanics, Volume I. North-Holland, 1976.

[4] Rudnicki, J. W. and J. R. Rice, Conditions for the localization of deformation in pressure-sensitive dilatant materials, Journal of the Mechanics and Physics of Solids, Vol. 23, 371-394, 1975.

[5] Wawersik, W. R., J. W. Rudnicki, W. A. Olsson, D. J. Holcomb and K. T. Chau, Localization of deformation in brittle rock: theoretical and laboratory investigations, in Micromechanics of Failure of Quasi-brittle Materials (ed. 
S. P. Shah. S. E. Swartz and M. L. Wang), Elsevier, New York, pp. 115-124, 1991.

[6] Press, W.H., B.P. Flannery, S.A. Teukolsky, and W.T. Vetterling, Numerical Recipes, The Art of Scientific Computing, Cambridge Univ. Press, New York, 1986

[7] Nelder, J.A. and R. Mead, Computer Journal, vol. 7, p. 308, 1965

[8] Olsson, W.A., The formation of a yield-surface vertex in rock, in Proc. $33^{\text {rd }}$ U.S. Symp. on Rock Mech., eds. J.R. Tillerson and W.R. Wawersik, pp. 701705, A.A. Balkema, Brookfield 


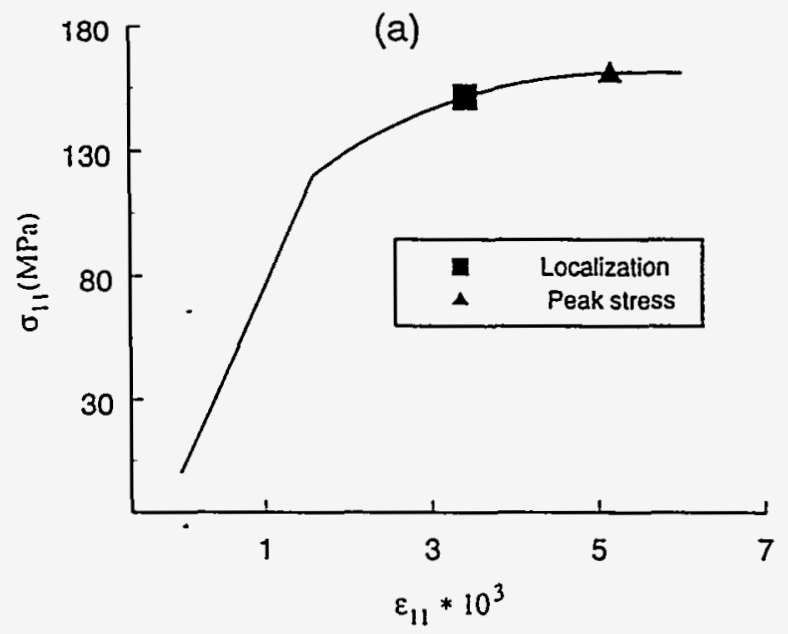

(b)
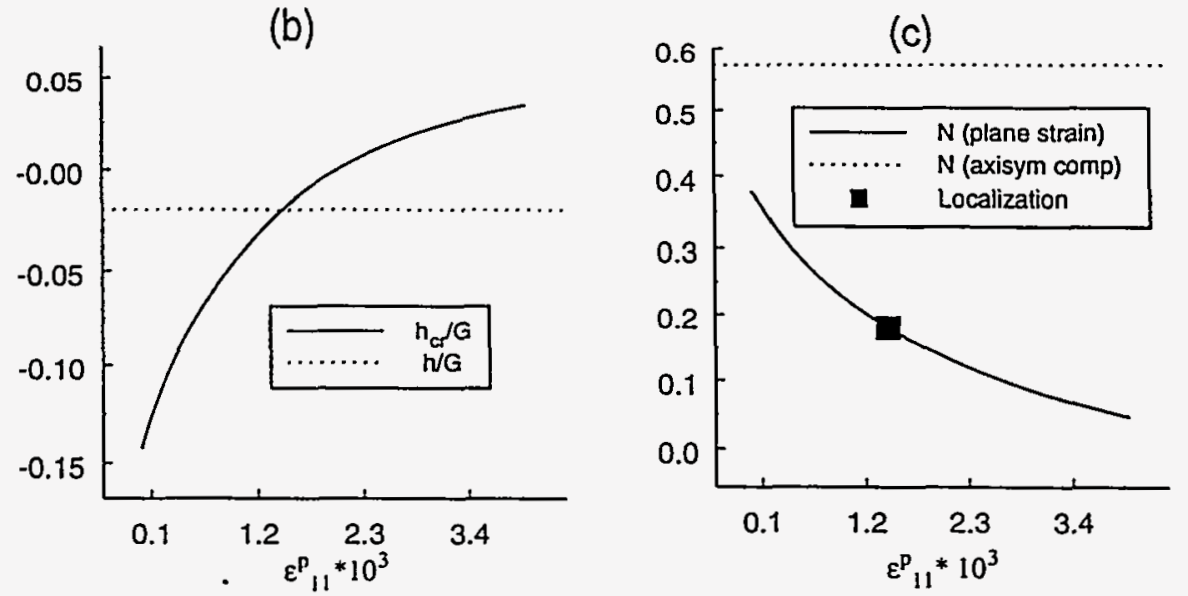

Figure 13: An example showing that $h$ can be negative at localization under plane strain conditions while the apparent hardening in positive. (a) Curve of axial stress $\left(\sigma_{11}\right)$ vs. axial strain $\left(e_{11}\right)$ for plane strain loading, continues to rise until the peak stress (triangle) even though $h / G=-0.02$. Localization (square) is predicted occur when $h_{c r} / G$ rises to equal $h / G=-0.02$. (b) Evolution of $h_{c r}$ is due to evolution of $\lambda^{\circ}(\mathrm{c})$ toward pure shear $(N=0)$. 